\section{DIVISION F COMMISSION 20}

\author{
POSITIONS \& MOTIONS OF \\ MINOR PLANETS, COMETS \& \\ SATELLITES \\ POSITIONS ET MOUVEMENTS \\ DES PETITES PLANÈTES, \\ DES COMĖTES, ET DES SATELLITES
}

\author{
PRESIDENT \\ VICE-PRESIDENT \\ SECRETARY \\ PAST PRESIDENT \\ ORGANIZING COMMITTEE
}

\author{
Steve Chesley \\ Daniela Lazzaro \\ Andrea Milani \\ Yoshikawa Makoto \\ Shinsuke Abe, Alan Gilmore, \\ Mikael Granvik, Robert Jedicke, \\ Petr Pravec, Tim Spahr, \\ Jana Tichá, Jin Zhu
}

\title{
TRIENNIAL REPORT
}

\section{Commission Activities in the Past Triennium}

This triennium has seen progress in a number of directions related to Commission 20 objectives. Foremost, the growth in the number of astrometric observations of small solar system bodies continues to accelerate and the total number of measurements recorded by the Minor Planet Center now exceeds 135 million. Currently the Pan-STARRS project and the Catalina Sky Survey (CSS) dominate detection and discovery efforts, while the NEO-WISE space mission contributes infrared detections valuable for understanding the size distribution of populations. Looking forward, the Large Synoptic Survey Telescope (LSST) is now funded and in construction on Cerro Pachon in Chile. LSST has the potential to revolutionize the field by conducting a multi-color, ten-year, all-sky survey with a limiting magnitude $\sim 24.5$ in the r-band. Survey operations are set to begin in 2022 .

The second Earth impactor reported prior to atmospheric entry was discovered in the first hours of 2014. Like the prior case of $2008 \mathrm{TC}_{3}, 2014 \mathrm{AA}$ was a few meters in size and was discovered $\sim 21$ hours prior to impact by R. Kowalski at the CSS survey telescope operating at Mount Lemmon in Arizona. Unlike $2008 \mathrm{TC}_{3}$, which impacted the desert in Sudan and yielded meteorites, 2014 AA was lost in the Atlantic Ocean. These two cases point to the likelihood that small impactors will continue to be discovered, affording an opportunity to obtain ground-truth information for calibrating asteroid physical observations. The forthcoming ATLAS survey now reaching operational capability may provide a more consistent source of such detections.

The most energetic asteroid impact since the Tunguska event occurred on 2013 February 15 near Chelyabinsk, Russia. The impactor was $\sim 20 \mathrm{~m}$ in size and delivered an energy of $\sim 500 \mathrm{kt}$ TNT equivalent. The impact led to nearly 1500 injuries, primarily from flying 
glass from windows shattered by the shock wave overpressure. In Chelyabinsk and nearby communities the damage was extensive, consisting of countless broken windows and the collapse of at least one building. Thus, while being smaller in energy than Tunguska, in terms of injuries and property damage the Chelyabinsk impact was undoubtedly the most destructive in recorded history. This event has re-energized international efforts to prevent or mitigate the impacts of asteroids and comets.

As the second phase of the reorganization of the IAU (the first being the reorganization of Divisions), all Commissions have been dissolved following the XXIX GA in Honolulu, and a newly formed suite of Commissions will carry forward the objectives and activities of the prior Commissions. In the case of Commission 20, the new cross-division Commission X2 on Solar System Ephemerides has accepted the mandate of Commission 20, as well as many of the responsibilities of the Commission 4 on Ephemerides. Commission $\mathrm{X} 2$ has equal standing in both Division A \& F.

This report is organized as follows. We shall first review the activities of the Commission during the past triennium and record the reports of associated Services and Working Groups for this period. This will follow with a retrospective look at the scientific and technical progress seen by Commission 20, from its founding in 1919 until its dissolution in 2015. We close with a report on the final business meeting of Commission 20, held in August 2015 at the XXIX General Assembly in Honolulu.

\subsection{Scientific Conferences}

During the triennium covered by this report, the following meetings were held that are of relevance to the work of Commission 20.

- Asteroids, Comets and Meteors 2014, 30 June-July 4 2014, Helsinki, Finland

- IAU Symposium 310: "Complex Planetary Systems," 7-11 July 2014, Namur, Belgium

- 4th IAA Planetary Defense Conference, 13-17 April 2015, Frascati, Italy

- IAU Symposium 318: "Asteroids: New Observations, New Models," 3-7 August 2015, Honolulu, Hawaii, USA

We note in particular the IAU Symposium 318 "Asteroids: New Observations, New Models," which was sponsored and primarily organized by Commission 20 and for which Commission 20 President S. Chesley served as Chair of the Scientific Organizing Committee. The meeting was held during the first week of the XXIX IAU General Assembly and included 99 contributions (18 Invited, 52 Contributed Oral and 29 Poster). The Symposium Proceedings will include 51 contributed papers, each of which was carefully reviewed or refereed, and should be available in early 2016.

\subsection{Astrometric Data Exchange Standard (S. Chesley)}

Commission 20 members played a key role in the development and establishment of the Astrometric Data Exchange Standard (ADES) to better facilitate the sharing and distribution of small body astrometric data. Before ADES, the standard format for exchanging astrometric data was derived from 80-column ASCII punch card records, a format formally known as MPC1992. The MPC1992 standard had numerous deficiencies in the modern era, including insufficient space for recording plain text numbers and designations, insufficient space for recording high-precision times and astrometric positions, lack of measurement uncertainty information, and lack of accessible context for the measurement reduction (e.g., star catalog employed, signal-to-noise ratio, etc.).

The new ADES version, provisionally dubbed IAU2015, removes the obstacles imposed by the 80 -column format and allows the observer to report additional information that is essential in assessing how the data should be used for fitting orbits (in the case of 
astrometry) or estimating absolute magnitudes (in the case of photometry). A key objective in developing ADES is to have a flexible packaging for the standard that could neglect fields that were not reported by the observer, and yet allow user-created fields that are not a part of the standard.

A key challenge seen early on was the need to serve two distinct user communities. Users who produce, maintain, and process the very large astrometric catalog (currently $>10^{8}$ records and perhaps $>10^{9}$ records within a decade) sought a packaging scheme that allows the data to be readily ingested, validated, and manipulated in modern computer systems, while users producing and using small data sets desired a plaintext format that can be readily edited and directly viewed. To serve both communities for the foreseeable future, a compromise was reached wherein XML was selected as the primary means of exchange, while a plain-text pipe-separated values (PSV) format will be available for those who require a more hands-on approach to preparing and viewing the data files. Under this scheme, the PSV format is compliant with the standard and can be used locally and shared with others. However, PSV must either be converted to XML before submission of observations to the MPC, or the observer must submit PSV through a web form that will perform the conversion as a part of the submission process.

The standard was initially developed in a community workshop held 7-8 May 2015 hosted by the Minor Planet Center at the Smithsonian Astrophysical Observatory in Cambridge, Mass. From there the standard continued to evolve under community input until a draft was released for broad public distribution via an MPC Editorial Notice (MPEC 2015-O06) that provided a detailed draft of the proposed ADES and invited community input. The draft ADES was endorsed by a vote of Commission 20 at the XXIX GA in Honolulu, with the understanding that minor revisions would be naturally be incorporated as the software development that is an essential element of the standard matures.

At the time of this report, the effort is underway to develop the publicly-distributed computer programs, scripts and libraries that the community will use to make the transition to ADES. We anticipate that by early 2016 the software will be widely distributed and that shortly thereafter the MPC will begin accepting and distributing ADES data in the IAU2015 format. The MPC will continue to accept and distribute the MPC1992 format during a 1-2 year transition period.

\subsection{Minor Planet Center \\ (G. Williams, Associate Director $\&$ M. Holman, Interim Director)}

\subsubsection{Introduction}

The activity of the Minor Planet Center (MPC) increased noticeably during the triennium. This report covers the period since the prior triennial report, specifically 2011 October 13 to 2015 September 28, and the use of the phrase "during the triennium" should be understood to mean this period. Increases are referred to the previous triennium.

During the triennium, the total number of observations in the MPC's files increased by $56 \%$, from 86.4 million to 135.2 million. The productive existing Pan-STARRS 1 (Panoramic Survey Telescope and Rapid Response System) was joined by an identical Pan-STARRS 2, while the Catalina Sky Survey and Mount Lemmon Survey continued to be very productive. The Siding Spring Survey, part of the whole Catalina Survey, was shut down in 2013. A new Lincoln (Laboratory) Near-Earth Asteroid Research (LINEAR) program, utilizing a 3.5-m reflector, has already produced 6.5 million observations, and the Wide-field Infrared Survey Explorer (WISE) continues to observe from low Earth orbit. 
The number of numbered minor planets increased by $48 \%$, from 301841 to 446782 , during the triennium. The number of a minor planet should signify that the object has been sufficiently well observed that it is unlikely to be lost in the foreseeable future. The numbered minor planets represented $53 \%$ of the orbits in the MPC files in 2011 October, and $64 \%$ in 2015 September.

\subsubsection{Publications and archiving}

The permanent archiving of data continues to be done on a monthly basis, coinciding with the publication of the Minor Planet Circulars (MPCs) and Minor Planet Circulars Orbit Supplement. As the traditional publication of the MPC, dating back to 1947, the former is a summary of MPC activity, the 19128 pages published during the triennium, a $43 \%$ increase, bringing the total to 95806 . The Orbit Supplement, first published in 2000, gives the full details on the new numberings and the new identifications, the 141014 pages published during the triennium (a 107\% increase) bringing the total to 349380 . The Minor Planet Circulars Observation Supplement, listing the bulk of the minor-planet observations, began publication in 1997. The 236968 pages published during the triennium (a $66 \%$ increase) brings the total to 631944 . All three permanent publications are now issued only in machine-readable form, via PDFs downloadable from the MPC website.

In addition, the Minor Planet Electronic Circulars (MPECs), first issued in 1993, provide immediate information on newly-designated NEOs, TNOs and comets. A total of 7811 MPECs were issued during the triennium (an increase of 27\%), bringing the total issued to 30 033. The 'Daily Orbit Update' (DOU) MPECs, prepared entirely automatically each night, tabulate all the orbits computed and identifications found during the previous 24 hours. Each DOU MPEC also includes continuing observations of all NEOs, the automatic preparation precluding the crediting of the observers in a reliable fashion. The DOU issue is consistent with the intention that the MPECs are a temporary publication.

A total recode of the MPC's processing pipeline is in the works, adopting the IAU ADES format for observations. It is anticipated that permanent archiving and publication of identified or newly-designated objects will occur several (eventually, many) times a day. More details on these changes will be forthcoming.

The MPC also hosts the Light Curve Database (LCDB), a repository of minor-planet light-curve observation files, on behalf of an outside group. The LCDB contains almost 2.5 million observations in 145388 separate data files representing 11452 different objects. The LCDB can be queried via the MPC website and observers can upload new data files via the $\mathrm{DB}$ interface.

The "NEOCP Blog" deals exclusively with the NEOCP and allows observers to send reports on what they are planning to observe, and what they actually observe. Posts on the blog are of a transitory nature. The "Daily Minor Planet Center" is intended for longer articles, written on a variety of topics by the MPC staff.

\subsubsection{Near-Earth objects}

Prior to the preparation of an $M P E C$ documenting a discovery, alerts to possible NEOS and comets are issued on the "NEO Confirmation Page". A number of functional updates to this page were made during the triennium.

During the triennium, 4792 separate NEOs were discovered, and of these 363 are considered Potentially Hazardous Asteroids, with a minimum orbit intersection distance with Earth less than 0.05 AU and absolute magnitude $\mathrm{H}$ brighter than 22. More than $99 \%$ of NEO discoveries were made by professional astronomers (over $99 \%$ of which were 
U.S.-based or U.S.-funded), although amateurs continue to provide follow-up astrometric observations of NEOs and NEO candidates.

The external alert system described in the previous triennial report was triggered at least eight times during this triennium.

The problem of confusion of high-altitude artificial satellites with natural objects, mentioned in the last triennial report, continues.

\subsubsection{Comets $\&$ distant objects}

Some of the NEO candidates appearing on the NEOCP turn out to be comets. The MPC worked in cooperation with the Central Bureau for Astronomical Telegrams (a service of Division B and Commission 6) in the announcement of new comets. Routine astrometric follow-up of comets was handled by the MPC. Almost 252000 comet observations were published during the triennium, bringing the total in the MPC archive to 847000.

As a result of Commission 6 not being renewed in the 2015 IAU Commission reorganization, the IAU delegated the MPC to handle the former Commission 6 tasks relating to the solar system (assigning provisional designations to comets and natural satellites, and assigning names of comets).

Although only 259 new 'distant objects' (centaurs and transneptunian objects) were discovered during the triennium, bringing the total to 1919 , more than $80 \%$ of those discoveries have been observed at more than one opposition.

\subsubsection{Outer satellites of the giant planets}

Because of their potential confusion with minor planets, the MPC continued to catalogue observations of and to compute orbits for the outer satellites of the giant planets. Although many astrometric observations of known objects were received during the triennium (and published in the Minor Planet Circulars), there were no discoveries reported. One Jovian satellite observed at the 2000 opposition was rediscovered on images taken in 2010 and 2011. Although this recovery was reported in 2012, the satellite was not numbered Jupiter LIII (Dia) until May 2015. At the same time, the numberings of Jupiter LI and Jupiter LII were also announced, both objects being unnamed.

\subsubsection{Personnel}

Former Director Timothy B. Spahr resigned on 2015 January 25. The Smithsonian Astrophysical Observatory, host of the MPC, appointed Matthew J. Holman as Interim Director shortly thereafter. Gareth V. Williams continues as Associate Director, with other staff members Michael Rudenko (returned from Harvard), Sonia Keys and José Luis Galache. Programmer James Davies was hired during the triennium, but left the MPC shortly after it ended.

\subsubsection{The end of the MPC under Commission 20}

Following the IAU Commission reorganization, Commission 20 ceased to exist at the Honolulu General Assembly. The MPC as a vital service will continue under the new organizational structure. It is sobering to consider that in the 68 years since the MPC was founded, the volume of observational actitvity has increased by more than three orders of magnitude. Yet the total number of people who have worked full time, processing observations and/or orbits, at the MPC is only nine: Paul Herget (1947-1978), Eugene Rabe (1948-1960s?), Conrad Bardwell (1958-1989), Brian Marsden (1978-2010), Daniel Green (1980-2000), Gareth Williams (1990-present), Timothy Spahr (2000-2015), Sonia Keys (2002-present), Michael Rudenko (2009-present) 


\subsection{Working Group on Natural Planetary Satellites (J.-E. Arlot)}

\subsubsection{Introduction}

The IAU has mandated a complete renewal of Commission and Working Group structures. So, it is time to report the activity of the Working Group on Natural Planetary Satellites, which was created in 1973 at the IAU General Assembly in Sydney. At the beginning, the working group was affiliated only with Commission 20 (Positions \& Motions of Minor Planets, Comets \& Satellites). The aim of the working group was to encourage observations and modeling of the motion of the natural planetary satellites. The first task was to gather astrometric observations which were not easily available. A database was proposed, gathering almost all published astrometric observations. Ephemerides were also provided and the working group became associated with Commission 4 (Ephemerides), Commission 8 (Astrometry) and Commission 7 (Celestial Mechanics). Later, it became affiliated with Commission 16 (Physical Study of Planets and Satellites), since both astrometric observations and dynamical modeling were depending on some physical parameters of the satellites.

At the present time, the working group maintains a database hosted by IMCCE (Paris observatory, France) and by Sternberg Astronomical Institute (Lomonosov University, Moscow, Russia).

At the time of preparing the present report, the membership of the Working Group is J.E. Arlot (President), M. Assafin, C. Blanco, J. Camargo, N. Emelianov, R.A. Jacobson, D. Pascu, Q.Y. Peng, M. Soma, P.K. Seidelmann, D.B. Taylor, R. Viera-Martins, and G. V. Williams.

\subsubsection{The new goals of the Working Group}

The WG decided to promote new goals as follows:

- The increase in astrometric accuracy of satellite observations

- The use of physical parameters to improve the dynamics of natural satellites and photometric data for the identification of the center of mass on the images

- The use of the future Gaia reference catalogue for a new reduction of old observations enabling a global reference system for all data used for satellite ephemeris purposes.

\subsubsection{The database maintained by the Working Group}

The WG maintained first a web site providing

- links to ephemeris services (IMCCE, JPL, MPC)

- bibliographic database of papers related to natural satellites

- tables of physical and dynamical parameters and links to NASA and USGS data on pole and rotation parameters

- software and miscellaneous data.

Then, the WG proposed a database of most of the published astrometric observations for each satellite system. The majority of the observations are either RA and DEC or relative positions to the planet or other satellites. For some main satellites, data on the observation of phenomena (eclipses by Jupiter, mutual events, occultations of stars) were provided.

The WG has the goal to upgrade the database into standard format. With support from the European Union, a new database providing standard data is now proposed. The standard format elaborated by Commission 20 for small bodies will have to be extended in order to be used for some of the satellite data.

Table 1 summarizes the contents of the WG database. Note that all of these observations are published observations with all the needed metadata. Other observations 
Table 1. Contents of Satellite Observation Database.

\begin{tabular}{lr}
\hline Satellite(s) & No. Observations \\
\hline Phobos, Deimos & nearly 5000 \\
Galilean & 12641 \\
Jupiter, inner & 1294 \\
Jupiter, irregular & 10820 \\
Saturn, inner & 1286 \\
Saturn, main & 51921 \\
Saturn, irregular & 5505 \\
Uranus, inner & 265 \\
Uranus, main & 5200 \\
Uranus, irregular & 790 \\
Triton & 4700 \\
Nereid & 900 \\
Neptune, other & 312 \\
Charon & 78 \\
Pluto, other & 42 \\
\hline \multicolumn{2}{c}{$\dagger-$ Some gathered by the MPC. }
\end{tabular}

are available, especially observations from space probes, which are not included in the database because of the lack of publication providing the information. The WG encourages the owners of observational data to publish them, making them available and useful for all.

\subsubsection{The impact of Gaia on the natural satellite ephemerides}

We are waiting for observations to be made by Gaia, which are expected to comprise about 50 observations of each satellite to magnitude 20 (except the Galileans and Titan, which are too large) made over a 5 -year period. These observations would be very accurate but limited to a restricted interval of time. Besides the observation of the satellites themselves, Gaia will provide a reference star catalogue of one billion stars (to magnitude 20) with an accuracy better than one mas, compared to the present catalogues (such as UCAC4) that provide an accuracy around 50 mas. Furthermore, the Gaia catalogue will provide proper motions of stars with an accuracy of one mas per century. Because of the advancement expected from Gaia we encourage the following activities:

- A new reduction of old data would be useful, not only to increase the accuracy of the positions, but also to place all observations in the same reference frame, which is essential for ephemeris purposes

- New reduction techniques should be proposed to have the benefit of such an accurate reference star catalogue

- Studies should be made on the photometric aspect of the surface of the largest satellites in order to be able to quantify the shift between the observed center of light of the objects and the center of figure, which may be supposed to be at the center of mass of the satellite and is most useful for dynamical studies.

\subsubsection{Toward the past}

The availabiliy of the Gaia reference star catalogue to reduce old observations will lead astronomers to search for old observations (e.g., photographic plates), to digitize them and to reduce them with modern methods. We encourage laboratories, institutes and observatories to work in that direction. However, the number of available plates is so large that we invite astronomers to cooperate in the choice of the best plates to be studied. 
Note that an old observation is any observation made before Gaia (photographic plates, CCD, space observations).

\subsubsection{The giant planets}

The giant planets are not easily observable for astrometric purposes. The atmosphere of Jupiter and the rings of Saturn make the center of mass difficult to identify. The best way to get their positions is to observe satellites (including space probes near planets) which are orbiting around the center of mass of the system. A large part of the database could be used for that purpose when the positions are provided in RA and DEC.

\subsubsection{The ephemeris servers}

Ephemerides of the Natural planetary satellites are provided by several web sites, including the following:

- The JPL server "Horizons" provides ephemerides for all satellites with all useful parameters derived from the ephemerides

- The IMCCE/SAI server "Multisat" provides ephemerides for all satellites with several parameters useful for observers, together with plotted configurations. Observers may easily get residuals for their observations directly on the web page.

- The MPC server provides ephemerides for irregular outer satellites that have been observed as asteroids by surveys dedicated mainly to small bodies.

The Working Group includes members involved in each of these ephemeris sites.

\subsubsection{Conclusion}

The working group wishes to continue its work, encouraging a) regular campaigns of observations, b) reduction of old data that are useful for dynamical modeling, and c) publication of astrometric observations, especially those from space probes, with all necessary metadata. For the forthcoming triennium, the Working Group on Natural Planetary Satellites should be initially affiliated with the new Commission X2 "Solar System Ephemerides."

\section{Retrospective on Commission 20}

The present Triennial Report is the final one from Commission 20. As a part of the reorganization of IAU Commissions, all Commissions have been dissolved as of August 2015. Some Commissions have been reconstituted with very little change, while others have not been renewed. In the case of Commission 20, it will functionally be replaced by the new Cross-Division Commission X2 (Solar System Ephemerides), which will have an enlarged mandate to include the motion of the major planets.

Given that this report marks the dissolution of Commission 20, it is fitting to take a retrospective view on the scientific and technological advances Commission 20 has seen since its founding. Commission 20 has its origin as one of the 32 "standing committees" (i.e., Commissions) appointed at the Brussels Conference of July 1919. Three years later, the original Commission 21 (Comètes/Comets) was merged into Commission 20, thus establishing the sphere of responsibility of Commission 20. Table 2 lists the 21 Presidents of Commission 20 over the 96 years since its founding. We note extended terms of service of the first five Presidents, until 1967, after which individuals served as President for a single triennium.

In the following subsections we present brief summaries of scientific and technical progress in a few relevant areas that are a part of the broad arena of the trajectories 
of small bodies in the solar system. The list of topics is not exhaustive, nor are the individual contributions comprehensive. Our intention is to describe in broad details a few of the more dramatic revolutions seen in the field since 1919.

\subsection{Changes in the manner and rate of small body discovery (G. Williams)}

Since the founding of Commission 20, there have been huge changes in the way that observers make and report observations. From 1919 to 1945, the minor planets were handled by the Astronomisches Rechen-Institut (ARI) in Dahlem, a suburb of Berlin, and publication of observations was scattered in numerous journals and observatory publications.

In 1919, the bulk of minor-planet (and comet) observations that were reported in the Astronomische Nachrichten $(A N)$ were made visually, using micrometers to measure the offsets in R.A. and Decl. of a moving object from one (or more) comparison stars. A number of independent measurements in each axis, made at slightly different times, would be combined into a single position for an intermediate time (producing, in effect, a mininormal place. When comparisons were made to more than one comparison star, more than one final position would be reported, sometimes for the same instant of observation. Typical consistencies in such multiple observations were $2^{\prime \prime}$ to $4^{\prime \prime}$ in each axis, using the comparison-star coordinates present in contemporary catalogues. When such positions are re-reduced with modern positions for those same comparison stars, the consistencies can often be improved to much better than $1^{\prime \prime}$. Photography was used primarily for discovery of new objects, with approximate positions being measured from the plates. A new series of rapid-announcement circulars, the Beobachtungs-Zirkulars (BZ), were issued by the $A N$ from 1919. A total of 33 new (old-style) provisional designations were assigned during the year, with an additional nine designations being assigned later. Twenty new numberings, mostly discovered by Max Wolf and Karl Reinmuth at Heidelberg, brought the total to 914 .

Table 2. List of Presidents of Commission 20.

\begin{tabular}{cll}
\hline Term of Service & Name & Country \\
\hline $1919-1938$ & Armin O. Leuschner & U.S.A./Germany \\
$1938-1948$ & Eugene Delporte & Belgium \\
$1948-1955$ & Dirk Brouwer & U.S.A./Netherlands \\
$1955-1961$ & Sylvain Arend & Belgium \\
$1961-1967$ & Paul Herget & U.S.A. \\
$1967-1970$ & Gleb A. Chebotarev & U.S.S.R. \\
$1970-1973$ & Frank K. Edmondson & U.S.A. \\
$1973-1976$ & Lubor Kresák & Czechoslovakia \\
$1976-1979$ & Brian G. Marsden & U.K. \\
$1979-1982$ & Grzegorz Sitarksi & Poland \\
$1982-1985$ & Elizabeth Roemer & U.S.A. \\
$1985-1988$ & Yoshihide Kozai & Japan \\
$1988-1991$ & Richard M. West & Germany \\
$1991-1994$ & Andrea Carusi & Italy \\
$1994-1997$ & Donald K. Yeomans & U.S.A. \\
$1997-2000$ & Hans Rickman & Sweden \\
$2000-2003$ & Edward Bowell & U.S.A. \\
$2003-2006$ & Giovanni Valsecchi & Italy \\
$2006-2009$ & Julio A. Fernandez & Uruguay \\
$2009-2012$ & Makoto Yoshikawa & Japan \\
$2012-2015$ & Steven R. Chesley & U.S.A. \\
\hline
\end{tabular}

NOTE - From 1967 it became customary for the Commission President to serve for one triennium, and, with few exceptions, for the Commission Vice-President to become the next President. 
Observational activity increased over the next twenty years, resulting in more discoveries. In 1925, 106 new provisional designations were assigned, and 22 were numbered. In 1926, the ARI began issuing RI Circulars $(R I)$, a similar publication to the BZ. By the 1930s, more than 300 new provisional designations were assigned and 30-40 objects numbered each year. Most observing programs were reporting approximate photographic positions. Typical telescopes used for discovery purposes were wide-field instruments, covering several degrees on a single plate, albeit with a poor plate scale and significant distortion near the plate edges. Deliberate follow-up of known objects would use longfocal-length refractors, with much smaller fields of view and much better plate scales. However, measurement of such plates was time-consuming as it was a manual process, and the accuracy of the derived positions was often limited by the poor comparison star coordinates used. Micrometer observations continued to be reported.

World War II caused considerable disruption to observing programs, notably the active program at Simeïs in the Crimea. The program at Turku, Finland, remained active throughout the war years. The numbers of new provisional designations assigned in 1944 and 1945 were 72 and 50, respectively, and a number of these were quickly identified with known numbered objects. The problem of distributing ephemerides meant that identifying objects could be tricky. Publication of the $A N$ became sporadic after 1943 and had ceased by the end of 1944. The RI continued to be published until April 1945, but later issues could not be distributed until after the war ended (the last few were republished on early MPCs). There were 1564 numbered minor planets in 1945, but more than 200 of the objects were effectively lost, either having been seen at one opposition or not having been seen in many years.

Following the disruption to the activities of the ARI caused by the war, the IAU decided to transfer the handling of the minor bodies to a newly-created Minor Planet Center (MPC). The MPC was founded in 1947 at the Cincinnati Observatory, under the direction of Paul Herget. An early task of the MPC was the creation of a single collection of minor-planet observations. Initially, this was intended to be complete only back to 1939, but has since been extended (albeit incompletely) back to 1801 . The MPC began issuing the Minor Planet Circulars (MPCs), on a sporadic publication schedule. While a handful of micrometric observations were received in the following years, most of the observations were obtained photographically.

Very few new numberings were made in the next twenty years, as efforts were concentrated on recovering the lost numbered objects. The observing program started in 1949 at the Goethe Link Observatory, Indiana, was crucial to this project. The McDonald Survey (Kuiper et al. 1958, Astrophys. J. Suppl. 3, 289) was the first of the big surveys and was a precursor to the 1960 Palomar-Leiden Survey (PLS; van Houten et al. 1970, Astron. Astrophys. Suppl. 2, 339). The PLS used the 1.2-m Schmidt at Palomar to observe a region of sky $12^{\circ} \times 18^{\circ}$ on multiple nights, in each of two months. The limiting magnitude was about $V \sim 20.5$. Over 2400 objects were detected, most of which were new, and orbits were determined for 1800 of them. A survey using a 0.4-m double astrograph was started at the Crimean Astrophysical Observatory in 1963. By 1965 there were 1726 numbered minor planets.

Photographic surveys intended specifically to search for Near-Earth Objects (NEOs) were began in 1973 by Eugene Shoemaker and Eleanor Helin, using the 0.46-m Palomar Schmidt (Helin et al. 1976, Bull. Amer. Astron. Soc. 8, 458). Only objects with anomalous motion were measured and reported, as it was not practical to measure the position of every object detected on the films.

The move of the Minor Planet Center from Cincinnati, OH, to Cambridge, MA, in 1978, under the direction of Brian Marsden, coincided with a noticeable increase in 
Table 3. Increase in number of numbered minor planets, number of observations per year, number of active observing sites and percentage of observations made by CCD.

\begin{tabular}{lcrcc}
\hline Year & Numbered & Obs. in Year & Sites & \% CCD \\
\hline 1970 & 1779 & 6971 & 60 & 0.0 \\
1975 & 1966 & 10118 & 83 & 0.0 \\
1980 & 2321 & 15782 & 90 & 0.0 \\
1985 & 3357 & 27397 & 158 & 2.3 \\
1990 & 4679 & 68647 & 134 & 18.3 \\
1995 & 6752 & 206858 & 166 & 67.8 \\
2000 & 19910 & 3099491 & 316 & 95.5 \\
2005 & 120437 & 7595588 & 366 & 99.985 \\
2010 & 257455 & 9690225 & 451 & 99.957 \\
$2015(*)$ & 446782 & 13872863 & 419 & 99.999 \\
\hline
\end{tabular}

$(*)$ - Not complete.

observational activity. The publication schedule of the MPCs was changed to regular monthly publication, initially on the first of each month, later on the date of the Full Moon.

More photographic surveys were begun: Edward Bowell used the 0.33-m 'Pluto' telescope at the Lowell Observatory starting in 1979; the 1981 U.K.-Caltech Asteroid Survey (Bus et al. 1982, NASA Tech. Memo. 85127, 53) utilized the 1.2-m Siding Spring Schmidt; the Palomar Asteroid and Comet Survey (Shoemaker et al. 1993, IAU Symposium 160: Asteroids, Comets, Meteors 1993, 269), using the 0.46-m Palomar Schmdit, commenced in 1983. Attempts were later made at Lowell Observatory to measure everything on the films obtained in the PACS.

An early adopter of the CCD was the Spacewatch project (Gehrels et al. 1986, Astron. J. 91, 1242). But it was not until the mid-1990s that they became large enough (and cheap enough) that they were of general use in surveying. In addition, the available astrometric catalogues had not improved substantially in depth or accuracy since the days of micrometric observation. Improvements in the accuracy of observations had to await the availability of modern catalogues, such as the USNO-A and -B, the UCAC and the URAT. The accuracy of modern survey observations ranges from $\sim 0^{\prime \prime} .5$ to $\sim 0^{\prime \prime} .2$, while dedicated follow-up sites are capable of accuracies below $0^{\prime \prime} .1$. The obvious advantages of CCD images are that they can be manipulated immediately by computer programs, and obtaining positions of every detection in an image is as difficult as obtaining one position. So it is now normal practice for observers to report every moving object in their images. This has had the effect of vastly increasing the rate at which observations are reported (the MPC typically receives more observations in a day around New Moon than it did in the whole of 1990) and at which objects are numbered. Table 3 indicates the increasing rate of detections and numbering of minor planets.

At the present time, more than 50000 new provisional designations are issued each year, and it is not unusual to number 4000 objects each month. Many amateurs have been able to contribute astrometry as a result of the availability of commercial CCDs. Numerous professional CCD-based surveys, with a primary goal of detecting NEOs, began operation between 1995 and the present. Limited space here precludes mentioning these modern surveys any further. While amateurs were able to discover many new minor planets in the mid- to late-1990s, the professional surveys have been covering enough sky for long enough that most of the minor planets that can get brighter than $V=19$ have already been discovered. The era of amateur discovery of minor planets may be drawing to an end. 


\subsection{Cometary nongravitational acceleration models (H. Rickman \& G.B. Valsecchi)}

It was known that the orbital motions of comets are influenced by nongravitational forces long before the IAU was founded. The first case in point was comet $2 \mathrm{P} /$ Encke in the early 19th century, because it proved impossible to account precisely for the times of its returns without including a secular deceleration of a nongravitational nature, which would make the comet arrive at perihelion slightly earlier than otherwise. Johann F. Encke himself (1823, Berliner Astr. Jahrb. f. d. J. 1826, 124) thought of a resisting medium in interplanetary space, while Friedrich W. Bessel (1836, Astr. Nachr. 13, 345) made the conjecture that comets expelling material in the sunward direction would experience an outward recoil force, and depending on whether this outgassing is stronger before or after perihelion, the net effect could be either an acceleration or a deceleration. With the lapse of time, different comets would prove to exhibit nongravitational effects in both senses, and this clearly favored Bessel's hypothesis.

It would nevertheless take a very long time before Bessel's idea of a jet force due to outgassing was developed into a physical theory for the cometary nucleus. The year was 1950, and once again, Encke's comet was the subject of the seminal paper written by Fred L. Whipple (1950, Astroph. J. 111, 375). While this paper is famous mainly for its identification of a solid, icy conglomerate nucleus as the source of the phenomena observed in comets, it is also worth noting that among the observations supporting such a picture, a central role is played by the measurements of the nongravitational effects in the orbital motions. In this regard, comet $2 \mathrm{P} /$ Encke is an extremely valuable source of information due to its short orbital period (3.3 years) and the long time series of accurate timings of its perihelion passages.

It is easy to see that a jet force that is slight compared to the perturbing accelerations by the planets is most easily revealed by its influence on the orbital energy, causing a secular increase or decrease of the comet's mean motion. Thus, to account for the nongravitational acceleration in orbital linkages for comets, the first approach was simply to add a secular change of the mean motion, whose value would follow as a best fit to the astrometric observations. The first great improvement to this practice was introduced by Brian G. Marsden and his colleagues Zdenek Sekanina and Donald K. Yeomans (1973, Astron. J. 78, 211). They introduced an acceleration term into the cometary equations of motion, expressed as a constant vector $\left(A_{1}, A_{2}, A_{3}\right)$ multiplied by a dimensionless function $g(r)$ of the heliocentric distance $r$. While the function is prescribed, the values of the $A_{i}$ parameters can be fitted to the astrometric data.

This has ever since been the standard model for cometary nongravitational accelerations. Its virtue lies in versatility rather than accuracy. The components of the constant vector signify those of the nongravitational acceleration at $r=1$ au in the radial, transverse (in the orbital plane), and normal directions, respectively. The physics of the model is contained in the $g(r)$ function, which is fitted to a simplified theory developed by Armand H. Delsemme and D. C. Miller (1971, Planet. Space Sci. 19, 1259) for the sublimation rate of $\mathrm{H}_{2} \mathrm{O}$ ice into vacuum under solar heating. Of course, the use of a constant value for $A_{1}$ effectively kills off the effect of the radial force component and is contrary to Bessel's hypothesis. Thus, the observed delay or advance in the perihelion times is mainly absorbed into the transverse $A_{2}$ component. This, in turn, may be physically interpreted in terms of a thermal lag in the outgassing from a rotating nucleus.

Other models were developed too. Whipple and Sekanina (1979, Astron. J. 84, 1894) imagined the $2 \mathrm{P} /$ Encke nucleus as a spinning, oblate spheroid with high latitude active spots in order to explain the fan-shaped coma of this comet. The effect of the jet force depends on the orientation of the spin axis in a more intricate way due to the 
localized activity, and the spin may change with time due to the associated torque. One might hence understand the secular decrease of the nongravitational effect in $2 \mathrm{P} /$ Encke. Over the following years, several other short-period comets were studied using a similar formalism.

However, much progress would be achieved in the 1980's by using thermal models of an outgassing nucleus to place constraints on the behavior of the jet force. Hans Rickman and Claude Froeschlé (1983, in Cometary Exploration, Vol. 3, Budapest Akad. Kiado, 109) showed for the case of $1 \mathrm{P} /$ Halley that the parameters of the standard model are anything but constant, even if the nucleus has an isotropic activity distribution. Further investigation would show that the actual behavior of the jet acceleration during the orbital motion of a comet is too complicated and depends on too many physical parameters to allow any simple generalization of the standard formula by Marsden et al. (op. cit.), which has hence survived in most cometary orbit determinations.

Nevertheless, the subsequent time saw many attempts to determine physical parameters of comet nuclei from orbital linkages using complicated models for the nongravitational acceleration. While such results may suffer from ambiguities when solving for correlated parameters and are anyway of very limited use in the general case, there was also a successful modeling in terms of the spin parameters of the nuclei (the orientational angles of the spin vector and the lag angle), introduced into orbit determinations by Grzegorz Sitarski (1990, Acta Astron. 40, 405) and his colleagues in the 1990's.

At the same time, there was also the realization of the importance of Bessel's idea. As Halley's comet returned to perihelion in 1986, several space missions targeted the close environment of the nucleus, and a good determination of its volume was achieved by Horst Uwe Keller et al. (1986, Nature 321, 320). In this connection, Rickman used physical modeling of the nucleus to estimate the jet force due to outgassing. By using the well known Gauss equations, he could derive the time integral of the nongravitational acceleration from the well established delay of this comet by four days at each perihelion, and combining the force and the acceleration, he obtained the mass of the $1 \mathrm{P} / \mathrm{Halley}$ nucleus and thereby its bulk density. While the discovery of a large porosity of a cometary nucleus was of great importance, the key issue here is that most of the perihelion delay turned out to result from the perihelion asymmetry of the gas production rate. Thus, the calculation of the lag angle (the $A_{2}$ effect) proved to be of less importance, and the perihelion asymmetry (the $A_{1}$ effect) came out as the main contributor. Most of the uncertainty in the density derived from the uncertainty over the shape of the gas production curve (Rickman 1989, Adv. Space Res. 9, 59).

Since the gas production curves of comets could not be theoretically predicted except in very crude terms, an important step was taken by Yeomans and his colleague Paul W. Chodas (1989, Astron. J. 98, 1083-1093), when they replaced the symmetric function $g(r)$ by an asymmetric function $g\left(r^{\prime}\right)$, using $r^{\prime}(t)=r(t-\tau)$, in the standard formula. They showed that a much improved orbit determination could thus be obtained for comet $6 \mathrm{P} / \mathrm{d}$ 'Arrest, which is known for its strongly asymmetric light curve, and that the best results corresponded to a value of $\tau$ in agreement with the observed shift of the light curve. Another important result from this time was the discovery by Michel C. Festou and his colleagues Rickman and Lars Kamél (1990, Nature 345, 235) of a statistical correlation between the perihelion delays of periodic comets and the perihelion asymmetries of their gas production curves. This all served to prove the importance of the gas production curves for interpreting or even predicting the nongravitational effects of periodic comets.

More recently, the modeling of the nongravitational acceleration along the lines introduced by Whipple and Sekanina (op. cit.) has been extended into a Rotating Jet Model focusing on a localized active area by the efforts of Sławomira Szutowicz (2000, Astron. 
Astrophys. 363, 323) and Steven Chesley (2002, Bull. Am. Astron. Soc. 34, 869) in the early 2000's. The mass determination technique using nongravitational effects has been further developed by Björn J.R. Davidsson and Pedro J. Gutiérrez in several papers devoted to different short-period comets, which were targets of planned or realized space missions (e.g., 2006, Icarus 180, 224). They used a Monte Carlo modeling of the activity distribution around a triaxial ellipsoidal nucleus along with its spin properties to find the best fit to both the complete set of nongravitational effects (including the shifts of perihelion longitude and longitude of the ascending node) and the shape of the gas production curve to derive masses and bulk densities for those comets. A very important outcome is the recent verification of their result for $67 \mathrm{P} / \mathrm{Churyumov-Gerasimenko-} \mathrm{the} \mathrm{target} \mathrm{of} \mathrm{the}$ Rosetta mission - by direct mass determination through the radio science investigation by Martin Pätzold and colleagues (see Sierks et al. 2015, Science 347, aaa1044), showing that the foundations of the physical modeling of nongravitational accelerations in comets are correct.

Finally, while the perihelion delays that are so evident for many short-period comets are inaccessible for long-period or new Oort Cloud comets, nongravitational accelerations have been measured in such comets too. An important fact is that a basically outward, radial jet force will decelerate the comet along its inward orbital branch, and this makes the real orbit more similar to a purely gravitational orbit with larger energy, i.e., a more hyperbolic original orbit before the entry into the planetary system. Therefore, as shown by Małgorzata Królikowska (2006, Acta Astron. 56, 385), the apparently hyperbolic original orbits of many comets contributing to the so-called Oort spike actually turn into elliptic orbits, when the nongravitational acceleration is taken into account. This is of importance when probing the structure of this distant comet reservoir by the energy distribution of the new comets, as also shown by Królikowska in later work with her colleague Piotr Dybczyński (e.g., 2011, Mon. Not. R. Astron. Soc. 416, 51). In that paper they also showed that a significant fraction of long-period comets with perihelion distance in excess of 3 au (15 out of 64 , in their case) exhibit detectable nongravitational forces; this somewhat surprising result suggests that super-volatiles contribute importantly to the outgassing.

\subsection{The Yarkovsky effect (S. Chesley)}

\subsubsection{Description and historical perspective}

Historically, asteroids were distinguished from comets by virtue of the lack of comae and, by extension, a presumed lack of nongravitational accelerations. However, in recent decades we have come to understand that as a rule asteroids do experience nongravitational accelerations - and related torques. These phenomena, related to photon recoil acceleration, are slight but in many cases detectable, and moreover they are of vital importance to the modern understanding of the dynamics of asteroids, both individually and as populations.

The Yarkovsky effect describes the subtle effect of anisotropic thermal emission from an asteroid surface due to rotation. An asteroid's evening terminator is warmer than the morning terminator, and thus thermal photons produce a slight excess in recoil acceleration in a direction towards the morning terminator. This diurnal variant of the Yarkovsky effect is effectively nulled if the asteroid spin axis is in the plane of the orbit, although in that case the far weaker seasonal variant is maximized. The net transverse acceleration induced from the thermal recoil acceleration serves to add energy and thus increase semimajor axis in the case of direct rotation, and conversely to decrease semimajor axis for retrograde rotators. This drift in semimajor axis leads to a quadratic-in-time runoff in the orbital anomaly of the asteroid, compared to the case without the Yarkovsky effect. 
Ivan O. Yarkovsky was a Russian civil engineer of Polish descent who worked on scientific problems in his spare time. Among other efforts, he privately published manuscripts in 1888 and 1901 that described the notion of thermal photon recoil acceleration on planets as a means for counteracting the effects of ether drag that was then assumed to be acting. Much later, Yarkovsky's effect was revived through an oblique reference by Estonian astronomer Ernst Öpik (1951, Proc. Roy. Irish Acad. A54, 165), who recalled in 1951 a pamphlet published more than 50 years earlier that was presumed lost. In 2006 the lost pamphlet of Yarkovsky was in fact recovered by historical astronomy sleuth George Beekman (2006, J. Hist. Astron. 37, 71), who has shed new light on Yarkovsky's history and work.

From 1951, the idea of the Yarkovsky effect appeared only sporadically in the literature, primarily from the Soviet Union and the United States, until the late 1990's when the crucial importance of the effect began to grow clear. The work of David Rubincam (e.g., 1995, J. Geophys. Res. 100, 1585; 1998, J. Geophys. Res. 103, 1725) and Paolo Farinella and David Vokrouhlický (1998, Icarus 132, 378; 1999, Science 283, 1507) was instrumental to the recognition of the importance of the Yarkovsky effect. From 2000, the literature has seen an explosion of research articles that combine to paint a much richer picture of asteroid dynamics than was previously envisioned. The present report provides a highly abridged summary of developments related to the Yarkovsky effect. See reviews by Bottke et al. (2006, Annu. Rev. Earth Planet. Sci. 34, 157) and Vokrouhlicky et al. (2015, in Asteroids IV, Univ. Arizona Press, p. 509) for a more comprehensive treatment than can be provided here.

\subsubsection{Dynamical Implications}

The action of the Yarkovsky effect leads to a steady drift inwards or outwards of an asteroid's orbit, which over millions of years causes main-belt asteroids to drift into mean motion and secular resonances and thereby evolve rapidly onto, e.g., planet crossing orbits. This simple notion up-ended the classical theory that held that collisions in the main asteroid belt injected fragments directly into the resonances, a notion that was at odds with other work, including the long cosmic ray exposure ages of meteorites and the low escaping velocities of collision fragments. Invoking Yarkovsky drift resolved these conundrums and allowed establishment or refinement of a number of theories, such as the delivery mechanism for near-Earth asteroids (Farinella and Vokrouhlický 1999, Science 283, 1507; Morbidelli and Vokrouhlický 2003, Icarus 163, 120), the sometimes peculiar shape of asteroid families in proper element space (Bottke et al. 2001, Science 294, 1693; Vokrouhlický et al. 2006, Icarus 182, 92), and the ages of asteroid families (Nesvorný and Bottke 2004, Icarus 170, 324; Vokrouhlický et al. 2006, Icarus 182, 118). The Yarkovsky effect is now firmly situated as a unifying element of our understanding of asteroid dynamics.

Adding to the richness of this picture is the interplay between the Yarkovsky and YORP effects. The Yarkovsky-O'Keefe-Radzievskii-Paddack (YORP) effect, refers to the radiative torques on an irregular body from both reflected and thermally re-emitted photons (Rubincam 2000, Icarus 148, 2). It can be considered as the rotational analog to the Yarkovsky effect, which affects translation. An extensive body of literature describes the importance of the YORP effect and how to model it (e.g., Nesvorný \& Vokrouhlický 2007, Astron. J., 134, 1750; Scheeres 2007, Icarus 188, 430; Breiter and Michalska 2008, MNRAS 388, 927). One of the consequences of the YORP effect is that spin rates evolve in a cyclic manner; as rates increase mass shedding can reverse the torques and lead to spin down and as rates decrease to very low angular momentum the body can enter a tumbling mode from which it can reemerge with an increasing spin rate (Pravec et al. 
2008, Icarus 197, 497). Current research suggests that these so-called YORP cycles tend to retard Yarkovsky drift because the asteroid rotation can alternate between retrograde and direct through YORP cycles (e.g., Bottke et al. 2015, Icarus 247, 191). Understanding this relationship between Yarkovsky and YORP is crucial for proper interpretation of some Yarkovsky-related theories, such as the age of asteroid families.

The Yarkovsky effect is also a confounding factor in the hazard assessment of potential Earth impactors. In some cases the Yarkovsky effect is instrumental in determining whether an impact can occur and if so what the probability of impact is. And yet, in most cases the Yarkovsky drift rate is not constrained, neither through orbital deviations nor from direct estimates of physical or rotational properties. Typically, even the sign of the drift is unknown. Davide Farnocchia and colleagues (2013, Icarus 224, 192) have tackled this problem in the case of (99942) Apophis, where Yarkovsky dominates the prediction of extreme scattering encounters, and David Vokroulický and collaborators have extended the analysis to include the effect of non-principal axis rotation (2015, Icarus $252,277)$. They conclude that the Apophis impact probability is currently dominated by a tiny keyhole in 2029 leading to an impact in 2068 with probability of order $10^{-5}$. Elsewhere, the question of an Earth impact by (29075) 1950 DA in the year 2880 is dominated by Yarkovsky uncertainty (Giorgini et al. 2002, Science 296, 132). Farnocchia \& Chesley (2014, Icarus 229, 321) performed a comprehensive analysis to infer an impact probability $10^{-4}-10^{-3}$ nearly nine centuries from now.

\subsubsection{Direct Detections}

Besides the wealth of theoretical work related to the Yarkovsky effect, there have been direct detections of this slight acceleration in the orbital motion of near-Earth asteroids. David Vokrouhlicky and collaborators (2000, Icarus 148, 118) made the earliest predictions for the direct detection of the Yarkovsky effect, including (6489) Golevka, which was the first asteroid to reveal the Yarkovsky effect (Chesley et al. 2003, Science $302,1739)$. Radar ranging measurements were crucial for the Golevka detection, while asteroid (152563) 1992 BF unambiguously indicated Yarkovsky drift from only optical observations, primarily a Palomar precovery in 1953 (Vokrouhlicky et al. 2008, AJ 135, 2336). The most recent report indicates a total of 42 asteroids with estimated Yarkovsky drift rates that exceed $3 \times$ the formal uncertainty (Chesley et al. 2015, Proc. IAU Symp. 318 , in press).

Of particular note, (101955) Bennu, which is the target of NASA's OSIRIS-REx asteroid sample return mission, has a rich enough data set that the Yarkovsky drift has 0.5\% precision. Steve Chesley and colleagues $(2014$, Icarus 235,5$)$ report that a joint analysis of Arecibo Observatory radar observations, Spitzer Space Telescope thermal observations and the Yarkovsky drift estimate allows an unambiguous estimate of the mass and bulk density of Bennu, the first such estimate not based on gravitational perturbations.

\subsection{Nonlinear orbit estimation and ephemeris prediction (A. Milani 86 G. Tommei)}

\subsubsection{Historical summary}

The orbit determination problem is fundamentally nonlinear, although in favorable situations it can be linearized. In 1809 C. F. Gauss solved the problem in two steps. First, by a preliminary orbit from a few observations $(\geqslant 3)$, a strongly nonlinear problem; the two approximations by Laplace and Gauss, assuming a 2-body motion between the observing times, both reduce the problem to an algebraic equation of degree 8 . Then Gauss computed predictions and residuals, linearized the dependency on orbital elements, and solved for a correction of the orbit as a linear least squares problem. If the recomputed residuals are still large, the procedure can be iterated and is called differential corrections. 
The matrix solving the linear problem is the covariance matrix, which at convergence can be used to define an approximation of the Probability Density Function (PDF) as a normal distribution with mean at the converged orbit. Moreover, this probability density could be propagated to the state at a different epoch, again in a linearized approximation.

It has always been clear that this paradigm could fail, even catastrophically, if the preliminary orbit is a poor approximation (because of insufficient observational data) and the problem is still strongly nonlinear even starting from there, but also if the orbit propagation is strongly nonlinear, e.g., as a consequence of a chaotic orbit with exponential increase of uncertainty. However, this paradigm remained very effective in astrodynamics, where the quasi-linearity is enforced through the design of space missions by assigning enough tracking resources; for small bodies in the solar system and double stars, few objects had to be handled at anytime and the observations could be properly spaced to guarantee both a good preliminary orbit and a well constrained least squares solution.

This paradigm started to fail with the transtion to digital astronomy in the 1990s: if the number $N$ of objects, mostly asteroids, observed each night is large (today many thousands and hundreds of thousands with the next generation surveys), and each night for each object there is a small number of observations (typically 3-5) over a short time span (1-2 hours), then the data may even contain observations of most objects over 3 separate nights, allowing one to compute a preliminary orbit, but the computational complexity of order $N^{3}$ forbids testing of all possible triples, unless a filter of lower computational complexity is used to preselect triples (Kubica et al. 2007, Icarus 189, 151). In astrodynamics a similar problem occurs in surveys for space debris, which is also a large population.

As the data rates skyrocketed in the 1990's, interest also grew in trying to predict possible impacts of natural objects, mostly near-Earth asteroids, on our planet. Because of close approaches these orbits are strongly chaotic and the orbit propagation rapidly becomes wildly nonlinear. The Gauss paradigm, which assumes that the problem is quasilinear, i.e., nonlinear but leading to convergent differential correction iterations, was applicable in this problem only for comparatively large impact probabilities. The only other approach known was the Monte Carlo method, in which the distribution is sampled randomly according to the normal PDF (either in the observations space or in the orbital elements space) and each sample orbit is propagated over the time up to the event to be investigated, such as a possible collision. An estimate of the probability is given by the fraction of samples with collision. This method is effective if the probability of the event, such as a collision, is larger than the inverse of the practical limit on the number of sampling orbits.

\subsubsection{Modern methods of nonlinear orbit determination}

Many people have worked in the last 25 years to try and find solutions to this problem, and we apologize if we cannot cite them all. We shall try to briefly describe the work led by two main research groups.

The group mostly based at the University of Pisa, Italy, concentrated on a method based on "testing multiple hypotheses", also called geometrical sampling, in which a single asteroid with an uncertain orbit is replaced by a swarm of Virtual Asteroids (VAs). The VAs are not selected at random but in a geometrically organized way such that the confidence region (where the probability of finding the asteroid in the orbital element space is significant) is covered by the neighborhoods of the VAs, each small enough to justify local linearization. 
If the time span of observations is very short, such as 1-2 days for a main belt asteroid, the observed angular positions and rates form a 4-dimensional vector, the attributable, which is well constrained. To the contrary, the other two coordinates, range $\rho$ and range rate $\dot{\rho}$, are either very poorly constrained or totally unknown. In this condition the VAs can be chosen by a geometrically regular sampling of the $(\rho, \dot{\rho})$-plane, limited to the admissible region where solar system orbits are possible (Milani et al. 2004, CMDA 90, $59)$.

When the observations extend to a somewhat longer time span, say 3 nights or more, the confidence region becomes elongated along a one dimensional smooth curve, the Line Of Variations (LOV), which can be computed by a modified differential corrections algorithm (Milani et al. 2005, A\&A 431, 729). Then the VAs can just be a regular sampling of this line. In both cases, propagation of the uncertainty to some later time is obtained by propagating the orbits of a finite set of VAs (typically a few thousand) and by interpolating among them on the smooth geometric object (surface or line). This is similar to the method of manifold dynamics, which is used for different purposes, in astrodynamics.

The group mostly based at the University of Helsinki, Finland, concentrated on "smart Monte Carlo", also called statistical ranging, by also exploiting the idea that most of the uncertainty is represented by a plane with coordinates given by the two distances $\rho_{1}, \rho_{2}$ at two different times corresponding to two attributables. Statistical ranging samples the probability distribution of the observation errors at two times and the distribution on the $\left(\rho_{1}, \rho_{2}\right)$-plane, selecting only solar system orbits, either with or without a priori information based on population models. In this way the algorithm obtains a sample of the probabilistic distribution in the orbital elements space, which can be propagated to the event to be predicted (Virtanen et al. 2001, Icarus 154, 412); this sample can be smaller than the one of a full Monte Carlo, although larger than the ones used in geometrical sampling.

Comparing the two classes of methods, statistical ranging can provide a very refined probabilistic model of the possible orbits if the number of randomly generated orbits is very large. Geometric sampling is in general much more efficient computationally, but this is obtained by assumptions on the geometry of the confidence region which might occasionally fail.

\subsubsection{The identification problem and the proposed solutions}

When the number $N$ of objects observed in each night is large, the challenge lies with the identification problem, namely which observation belongs to which physical asteroid. Once enough observations belonging to a single object have been assembled, a good orbit can be computed and the problem of attributing additional observations to the same asteroid becomes quasi-linear. At the beginning, when no orbit is available, the problem is much more difficult, because it is strongly nonlinear and the solution algorithms have to be applied a large number of times.

To start the process, it is possible to sample the confidence region representing the possible orbits with insufficient observational information, e.g., corresponding to only 1-2 nights. If the number of VAs used to sample is much smaller than $N$, each VA can be propagated to cover the time span of successive observations, and still the computational complexity can be controlled between $N \log N$ and $N^{2}$ (Milani \& Gronchi 2010, Theory of Orbit Determination, Cambridge Univ. Press, Chap. 11).

Another class of methods uses the approximation that the orbit over the time span of the available observations is well approximated by a 2-body solution, and thus uses the constant values of the keplerian integrals (angular momentum, energy and the Lenz 
vector) to provide equations to be satisfied by either 2 or 3 attributables (Gronchi et al. 2015, CMDA 123, 1). These equations are converted exactly into algebraic equations in one distance, which have degree 8 (as in the Laplace-Gauss preliminary orbits). However, it is possible to build a sequence of gradual identifications getting to a well determined orbit with computational complexity of the order of $N^{2}$.

The methods above have not yet been used in a large scale attempt to solve, or at least mitigate, the problem posed by the $>10$ million observations stored at the Minor Planet Center which have not yet been used in an identification. This job should be undertaken in the next few years.

\subsubsection{The Impact Monitoring problem}

For impacts of comparatively large asteroids on the Earth we are interested in detecting Virtual Impactors (VIs), that is patches in the orbital elements space corresponding to collisions at some future epoch, even when the impact probability is small, say $10^{-6}$ or less. Thus the Monte Carlo methods are too slow. They can be used to solve the problem for a single asteroid, but not to maintain a comprehensive impact monitoring system, in which all asteroids are scanned for VIs in the next $\sim 100$ years whenever new observations are reported.

Thus starting from 1999 automated impact monitoring systems were operational, first NEODyS at University of Pisa and then in 2002 Sentry at NASA's Jet Propulsion Laboratory. Although the two software system were developed independently, they both use versions of the LOV algorithm to sample a few thousand VAs, propagate them for $\sim 100$ years into the future and then use interpolation along the LOV to fine tune the initial conditions and find a representative of the VI, that is an initial condition leading to impact (Milani et al. 2005, Icarus 173, 362).

Given the extreme chaoticity of the planet-crossing orbits, it is not possible to find in a deterministic way all the VIs, but it is possible to select a sampling providing reliable detection above a given level of impact probability. Currently this generic completeness is set at few parts in $10^{-7}$, with lower probability VIs being detected only probabilistically. Attempts to extend the time interval monitored for impacts to $\sim 200$ years have been done, but they require more sophisticated dynamical models, including non-gravitational perturbations, especially the Yarkovsky effect (Spoto et al. 2014, A\&A 572, A100).

Recently there has been a research effort to handle the case of imminent impactors, i.e., the asteroids just discovered and still with very few observations (often less than an hour arc), which have an essentially undetermined orbit, but could impact the Earth very soon (hours to weeks). The problem is difficult because the observations are so few that error models fail because of small number statistics. The solution could be found by using population models as a priori constriants (Farnocchia et al. 2015, Icarus 258, 18) or by using specific observer-supplied information on the quality of individual observations, as will be made possible by the new Astrometric Data Exchange Standard adopted by Commission 20.

\subsection{Near-Earth Objects (D. K. Yeomans)}

It is only relatively recently that the serious threat from near-Earth objects ${ }^{1}$ (NEOs) has been recognized. It has been more than a century since the discovery of the first NEO, (433) Eros, in 1898, and the first asteroid found on an Earth crossing orbit was (1862) Apollo in 1932. And yet it was not until the mid-twentieth century that lunar craters and

\footnotetext{
NEOs are comets or asteroids whose perihelia are $1.3 \mathrm{au}$ or less. The vast majority of NEOs are near-Earth asteroids (NEAs).
} 
Arizona's Meteor Crater were generally considered asteroid impact features, rather than being formed by volcanic activity (Baldwin 1949, The Face of the Moon, Univ. Chicago Press; Shoemaker 1963, in The Moon, Meteorites and Comets, Univ. Arizona Press, p. 301). At the beginning of 1950, there were only thirteen near-Earth asteroids discovered, five of which were so-called Potentially Hazardous Asteroids, or PHAs ${ }^{2}$.

\subsubsection{Organized searches for NEOs}

In the 1970's and 1980's, the first organized photographic searches for NEOs were carried out by Eleanor Helin, Gene and Carolyn Shoemaker, Tom Gehrels and Bob McMillan. Beginning in 1973, Helin and Gene Shoemaker began using the 18-inch Palomar Schmidt telescope in southern California to hunt for NEOs and Carolyn Shoemaker joined them in 1980. Progress was slow and by the beginning of 1980, there were still only 52 discovered NEAs, 17 of which were PHAs. In 1983, Gehrels and McMillan began using the 0.9-meter aperture Steward Observatory (Spacewatch) telescope near Tucson Arizona for NEO searches and by 1984, this telescope was dedicated full time for NEO searches using, for the first time, charge coupled device (CCD) detectors. The use of CCD detectors and large aperture, wide field telescopes dramatically increased the NEO detection rates. The Jet Propulsion Laboratory (JPL) Near-Earth Asteroid Tracking (NEAT) program, initially in cooperation with the Air Force, ran a NEO discovery program (1995-2007). The Lowell Observatory NEO Survey (LONEOS) utilized a 0.6-meter Schmidt telescope for NEO searches (1993-2008). The Catalina Sky Survey (CSS), near Tucson Arizona, began operations in 1993 and in late 2015 (when this summary was written), it is still one of the most productive NEO search efforts. For several years prior to it being retired in 2013, the 0.5 meter Uppsala Schmidt telescope at Siding Spring, Australia was operated by the CSS when it was then the only NEO search telescope operational in the southern hemisphere. Over the interval 1996-2013, the Lincoln Near-Earth Asteroid Research (LINEAR) program operated two, then one, 1-meter aperture telescopes near Socorro, New Mexico. The LINEAR program discovered the majority of the NEAs larger than one kilometer in diameter.

The Panoramic Survey Telescope and Rapid Response System (PAN-STARRS), which operates a 1.8-meter, wide field discovery telescope atop Haleakala on island of Maui in Hawaii, began NEO discoveries in 2010. The NEOWISE program observes NEOs in the near infrared wavelengths using the 0.4-meter telescope aboard the Wide-field Infrared Survey Explorer (WISE) spacecraft, which is in an Earth polar orbit. The NEOWISE program operated for ten months in 2010 before its cryogen ran out. It then ran an additional four months with only two of the four original infrared bands operational. After more than two and a half years of spacecraft hibernation, the NEOWISE program was re-activated in September 2013 for an additional 3-year planned observing period.

The ongoing CSS, Pan-STARRS and NEOWISE NEO discovery surveys provide the vast majority of current discoveries. The recent (2001-2015) impressive NEO discovery rates are evident from the number of discoveries by the beginning of 1990 (182), 1995 (369), 2000 (937), 2005 (3221), 2010 (6732) and 2015 (12057). It took over 100 years, from the year 1898 to 2000, to find the first 1000 NEOs. The current discovery rate is over 1000 NEOs per year.

A significant amount of observational, orbital, and ephemeris data are available from the IAU-sanctioned, and NASA-supported, Minor Planet Center (MPC) in Cambridge, Massachusetts. The MPC acts as the clearinghouse for international observations of all

\footnotetext{
${ }^{2}$ PHAs are asteroids whose absolute magnitudes are 22 or less and whose orbits can bring them to within 0.05 au of the Earth's orbit.
} 
asteroids and comets. Up-to-date orbital data, Earth impact probabilities and ephemeris data are available from the ESA-sponsored NEODyS site (http://newton.dm.unipi. it/neodys/) and from NASA's Center for NEO Studies at JPL (http://neo.jpl .nasa. gov). While he was the IAU General Secretary, Karel A. van der Hucht compiled an extensive chronological listing of NEO close approaches and significant events (http://www.iau.org/public/themes/neo/nea/).

\subsubsection{The changing public perception of NEOs}

The issue of the NEO threat was highlighted in 1980 when Walter Alvarez and colleagues (1980, Science 208, 1095) suggested that the transition between the Cretaceous and Tertiary eras was marked by the collision of a ten-kilometer sized NEO some 65 million years ago. This suggestion received solid support eleven years later when an impact crater of the appropriate size and age was discovered near Chicxulub Mexico (Hildebrand et al. 1991, Geology 19, 867). Media attention was also focused on the NEO threat issue when the Hubble Space telescope and other instrumentation observed the collision of about two dozen fragments of comet Shoemaker-Levy 9 with Jupiter in 1994.

The dramatic increase in NEO discoveries beginning in the late 1990's, and the increasing number of predicted Earth close approaches by these NEOs, were often broadcast by the media. While some of these media announcements were sensationalized and inaccurate, the media attention did sensitize the public and political leaders to the NEO threat issue. In 1994, the U.S. Congress asked NASA to report upon a program to identify and catalog, within ten years, all NEOs larger than one kilometer. In 1998, NASA formed its Near-Earth Object Observations Program to coordinate its NEO search and physical characterization efforts. The stated goal was to find $90 \%$ of the NEOs larger than one kilometer. In 2005, the U.S. Congress requested that NASA increase its efforts and find $90 \%$ of the NEOs larger than 140 meters. The successfully predicted impact of a 4-meter sized asteroid $\left(2008 \mathrm{TC}_{3}\right)$ over northern Sudan on October 7, 2008 (Jenniskens et al. 2009, Nature 458,485) and the unpredicted atmospheric impact of an 18 $\mathrm{m}$ asteroid near the Russian city of Chelyabinsk on February 15, 2013 dramatically underscored the asteroid threat as an international issue (Brown et al. 2013, Nature 503, 238; http://neo.jpl.nasa.gov/news/fireball_130301.html).

\subsubsection{The Internationalization of the NEO Program}

While NASA should be given credit for underwriting most of the past and present NEO detection, astrometric follow-up and characterization activities, a number of important international activities were carried out as well. For example, in 2008, the European Space Agency (ESA) initiated its Space Situational Awareness (SSA) program that included the study of NEOs along with space surveillance and space weather activities. ESA's NEO Coordination Centre has been established in Frascati, Italy and the ESA one-meter telescope on Tenerife has been used to provide NEO observations. Beginning in 2012, the European Union provided support for an international collaboration called NEOShield to investigate the prevention of impacts by asteroids and comets. These activities include investigations studying the deflection of an Earth threatening asteroid using a spacecraft impact technique.

Beginning in 2005, an Action Team of the Scientific and Technical Subcommittee under the UN-Committee On the Peaceful Use of Outer Space (UN-COPUOS) began discussions on the appropriate international responses to a NEO threat. Ten years later, this Action Team was dissolved after the successful formation of the International Asteroid Warning Network (IAWN) and the Space Mission Planning Advisory Group (SMPAG). 
The International Asteroid Warning Network (IAWN) was established in 2013 to create an international group of organizations involved in detecting, tracking, and characterizing NEOs. The IAWN is tasked with developing a strategy using well-defined communication plans and protocols to assist governments in the analysis of asteroid impact consequences and in the planning of mitigation responses (http://www.minorplanetcenter.net/ IAWN/).

The Space Mission Planning advisory Group (SMPAG) was established in 2013 by Member States of the United Nations that have space agencies. This group includes representatives of spacefaring nations and other relevant entities. Its responsibilities include laying out the framework, timeline and options for initiating and executing space mission response activities. The group will also promote opportunities for international collaboration on research and techniques for NEO deflection

(http://www. cosmos.esa.int/web/smpag/meetings).

\subsection{Trans-Neptunian objects (H. Rickman \& G.B. Valsecchi)}

For a long time, Pluto was the only known trans-Neptunian object and was counted with the planets. Hence it was outside the remit of IAU Comm. 20. However, from the time of its discovery in 1930, there were thoughts and speculations about many more objects existing beyond the orbit of Neptune.

The first sign of a change was the discovery of (2060) Chiron in 1977. Although its orbit is currently contained within the mean distance of Uranus, at the time of discovery it was in the outer parts of its orbit, and it was clear that it could signal the existence of many small objects in this region and beyond. This was related to the problem to account for the source of short-period comets - in particular, those of the Jupiter Family. Although Edgar Everhart (1972, Astrophys. Lett 10, 131) had shown that Oort Cloud comets with low inclinations and perihelia near Jupiter's orbit were particularly vulnerable to capture into the Jupiter family, doubts remained over the efficiency of this source.

An important result was achieved by Julio Fernández and Wing-Huen Ip (1983, in Asteroids, Comets, Meteors, Uppsala Obs., p. 387), who argued that the proper source was a "cometary belt" - a reminiscence of the planetesimal disk associated with the formation of Uranus and Neptune. The need for a low-inclination source was later verified by Martin Duncan and colleagues (1988, Astrophys. J. 328, L69), who coined the term "Kuiper Belt" for the source of Jupiter Family comets.

However, although searches were already ongoing, the observational verification of the Kuiper Belt would not occur until 1992 with the discovery of (15760) 1992 QB $_{1}$ by David Jewitt and Jane Luu (1992, IAU Circular 5611). With this discovery, Pluto was no longer the only trans-Neptunian object in the solar system, and as time went on, many more discoveries would follow, with the count now approaching 1500 objects. Thus it was clear that the solar system had indeed a new belt beyond the major planets, possibly outnumbering the asteroid belt by a large factor.

What is nowadays called the Kuiper Belt or the Edgeworth-Kuiper Belt is a vast population of objects whose perihelia are situated far beyond the orbit of Neptune, so that they are not immediately subject to close encounters with this planet. The largest members so far discovered are (136472) Makemake and (136108) Haumea, although the perihelion distance of the latter is a bit too small for this classification. Both objects are larger than (1) Ceres and are counted with (134340) Pluto as "dwarf planets." However, the total mass of this structure is less than $10 \%$ of the Earth's mass, so it is insignificant compared with the masses of the planets. The same holds for the sub-population called 
"plutinos" in 2:3 mean motion resonance with Neptune, for which Pluto is the namesake. The status of the latter into that of dwarf planet changed at the XXVII IAU General Assembly (2006), when it was decided to adopt a formal definition, partly based on dynamics, for solar system planets. As a consequence, the collection and archiving of the astrometric data for Pluto came under the responsibility of the Minor Planet Center, definitely improving their maintenance and availability, thanks to the experience and dedication of the MPC in these matters. This was accompanied by a renewed interest for the study of the dynamics of minor bodies within and beyond the region of the outer planets.

In 1996, the discovery of (15874) $1996 \mathrm{TL}_{66}$ (Luu et al. 1997, Nature 387, 573) showed that there exists another population of trans-Neptunian objects, whose orbits are more directly coupled to Neptune's orbit and often extend much further away from the Sun. This has since been termed the scattered disk. By mass, it does not dominate over the Kuiper Belt, but its largest member, (136199) Eris, is about as large as Pluto, and more objects of similar size may still await discovery.

Because of the closer link to Neptune, the orbits of scattered disk objects are unstable on a shorter time scale than those of Kuiper Belt objects. Hence, given that the population sizes are not widely different, the scattered disk was recognized by Martin Duncan and Hal Levison (1997, Science 276, 1670-1672) to be the major source of Jupiter Family comets. Its relative instability also shows that it may have been much more numerous and massive in the distant past, and it has come to be an important element in recent theories for the formation of the Oort Cloud of comets. Moreover, the so-called Centaurs, of which (2060) Chiron was the first to be discovered, are recognized as a transitory stage between the trans-Neptunians (mainly, the scattered disk) and the Jupiter Family - a fact that reflects an idea put forward by Elena Kazimirchak-Polonskaya (1972, IAU Symp. 45, p. 373) about the giant planets gradually transforming the orbits of comets between the Jupiter Family kind and more distant orbits.

The understanding of these dynamical paths has caused a shift of emphasis in the way Jupiter family and Halley type comets (JFCs and HTCs, respectively) are classified; traditionally, the distinction has been based on the orbital period $P: P<20$ yr for JFCs, and $20<P<200 \mathrm{yr}$ for HTCs. In view of the prevailing role of Jupiter in modifying the orbits of periodic comets, a new classification has emerged, based on the value of the near invariant parameter introduced by Félix Tisserand in the late 19th century: adopting as unit of length the semimajor axis of the orbit of Jupiter, JFCs are characterized by Tisserand parameter $T$ such that $2<T<3$, while HTCs have $T<2$ (Carusi et al. 1987 , A\&A 187, 899). The advantage of this classification is that less that $10 \%$ are transferred from one to the other dynamical group over long time scales, and most of those that change tend to remain near the Tisserand dividing line all the time (Levison \& Duncan 1994, Icarus 108, 18).

The motions of Centaurs are dominated by close encounters with the giant planets, which can induce large variations of the orbital semimajor axis. However, the evolution can slow when a Centaur orbit decouples from a planet and evolves only under the action of secular perturbations. This slow evolution continues until an orbital coupling is re-established with one or more planets, thus allowing encounters that restore the chaoticity of the motion. This overall pattern is characteristic of all bodies in planet crossing orbits, be they near Earth asteroids, Jupiter family and Halley type comets, or Centaurs.

The existence of the Oort Cloud was advocated by Jan Hendrik Oort (1950, Bull. Astron. Inst. Netherl. 11, 91) based on the orbits of long-period comets. It is best 
imagined as a nearly spherical envelope of the solar system at distances ranging from about $10000 \mathrm{au}$ to about ten times this distance. While the scattered disk may dominate as the source of Jupiter Family comets, the Oort Cloud likely dominates as the source of all other kinds of observed comets. Its origin remains an unsolved problem, like that of the scattered disk. In the framework of the Nice Model for the long-term evolution of the solar system, put forward by Kleomenis Tsiganis and colleagues (2005, Nature 435, 459 ), both structures may have arisen from a primordial disk of icy planetesimals beyond a tight system of initial giant planet orbits in connection with a dynamical instability of these planets, which likely occurred about 4 Gy ago.

Concerning the motions of comets in the cloud, Oort had originally envisaged that it would be dominated by the perturbations due to passing stars. However, John Byl (1983, Moon \& Planets 29, 121) showed that galactic perturbations would significantly affect the near-parabolic orbits of cloud comets; other studies followed along the same lines, leading to a situation in which the role of passing stars appeared to be rather unimportant. This was reversed by Hans Rickman and collaborators (2008, CMDA 102, 111), who found that stars play a fundamental role in the injection of comets into observable orbits, acting in synergy with the galactic tide, so that the injection rate due to the combination of the two is significantly larger than the sum of the two separate rates. Another important role is played by the planets, as shown by Nethan Kaib and Thomas Quinn (2009, Science $325,1234)$; in fact, encounters with the outermost planets can perturb comets in the inner part of the cloud, where they are less subject to galactic and stellar perturbations, transferring these comets to the outer part of the cloud, thus replenishing the source region of the observed cometary flux.

In 2003, a new class of trans-Neptunians was revealed, as Michael Brown, Chadwick Trujillo and David Rabinowitz discovered (90377) Sedna. With a semi-major axis close to $500 \mathrm{au}$, its perihelion distance of 76 au makes its orbit completely detached from the planetary system. This requires a mechanism of detachment, which may be the same that also created the Oort Cloud. In order to efficiently raise the perihelia of comets with such small semi-major axes, a dense stellar environment is necessary. In this case, close enough, slow encounters with other stars are likely, and angular momentum is easily added to the comet orbits.

Hence, the Sun's birth cluster should have been rich in stars - a conclusion reached by Fred Adams (2010, Annu. Rev. Astron. Astrophys. 48, 47) based on various evidence including the abundance of short-lived isotopes such as ${ }^{26} \mathrm{Al}$ in the solar nebula. Regarding the structure of the Oort Cloud, it is clear that the large distances from which the new comets are arriving represent only an outer halo, while an inner core may in fact be the heart and initial feature of this population.

In 2013, Ramon Brasser and Alessandro Morbidelli (2013, Icarus 225, 40) put forward a numerical model for the associated formation of the scattered disk and the Oort Cloud in the framework of the Nice Model. According to their results, the scattered disk was initially about 100 times more massive than today, and less than $10 \%$ of those objects found their way into the Oort Cloud by the action of galactic tides and passing stars of the galactic field. While this may possibly be consistent with the numbers derived from present-day observations, there are still issues about several factors. In particular, if the Sun escaped from its birth cluster only after the planetary instability, the Oort Cloud may have been formed in a rich stellar environment as a compact, massive feature. Sedna may be a survivor of this initial cloud, while the currently active outer halo was formed later by outward diffusion, as implied by the models of Marc Fouchard and colleagues (2014, Icarus 231, 99). 


\section{Business Meeting (A. Milani)}

The triennial meeting of Commission 20 was held at the XXIX GA starting August 4, 2015 at 8:30, and continued in a second session on August 10, 2015 from 15:00.

After the welcome from the President S. Chesley, three reports were presented (they are summarized in Section 1).

(a) Status and plans for the Minor Planet Center (G. Williams);

(b) Working Group on Natural Planetary Satellites (J.-E. Arlot);

(c) IAU Symposium 318 "Asteroids: New Observations, New Models" (S. Chesley).

On point 1., a vote was held on what should be the IAU body responsible for the MPC service; with large majority it was decided that this should be the new Commission X2 "Solar System Ephemerides".

The new initiative undertaken by the Commission on the establishment of a new Astrometric Data Exchange Standard (ADES) was illustrated by D. Farnocchia. After preparatory work started in August 2014 (by Chesley, Farnocchia, Milani and Spoto) a proposal was presented and widely discussed in a meeting hosted by the MPC May 7-8, 2015. Given the progress done in that meeting, a final version was composed and disseminated by MPEC Editorial Notice in July 2015. There was an ample discussion on several technical points, but overall good agreement. Thus the ADES was submitted to vote for official adoption by the Commission (proposed by MacMillan, seconded by Valsecchi), and approved unanimously (with 1 abstention): thus the new standard has been officially adopted by Commission 20 .

A new Asteroid Light Curve Data Format (ALDEF) was presented by B. Warner; these data should be stored at and made public by the MPC.

The President announces that the Commission should present a report for the IAU Transactions A, and that he intends this to be more of the nature of a legacy report, presenting the evolution of the field over several years, rather than the usual triennial report. Manuscripts of contributions to this are due by October 31, 2015 (they are inserted in this publication, as subsections of Section 2).

The President reads the list of Commission 20 members deceased recently: Y. Batrakov, G. Blow, J. Eliott, P. Message, G. Sitarski, I. van Houten-Groeneveld. One minute of silence is observed to honor their passing.

Information on the process of transition, between Commissions 20 and 4, and the new Commission X2, is given by A. Milani.

A discussion is held on the best choices to propose IAU Symposia for the next triennium. A proposal for the year 2017 would need to be put together very soon. Given the success of the IAUS 318, also on increasing the visibility of the astronomical disciplines of interest for Commission 20 to the entire Union, the Commission proposes (to the future Commission X2) to apply for the organization of a Symposium to be held at the XXX General Assembly in Vienna. 